CUBO A Mathematical Journal

Vol.21, № 03, (09-27). December 2019

\title{
Naturality and definability II
}

\author{
Wilfrid Hodges ${ }^{1}$ and Saharon Shelah ${ }^{2}$ \\ ${ }^{1}$ Herons Brook, Sticklepath, \\ Devon EX20 2PY, England. \\ wilfrid.hodges@btinternet.com \\ ${ }^{2}$ Institute of Mathematics, Hebrew University, \\ Jerusalem, Israel. \\ shelah@math.huji.ac.il
}

\begin{abstract}
We regard an algebraic construction as a set-theoretically defined map taking structures $A$ to structures $B$ which have $A$ as a distinguished part, in such a way that any isomorphism from $A$ to $A^{\prime}$ lifts to an isomorphism from $B$ to $B^{\prime}$. In general the construction defines $B$ up to isomorphism over $A$. A construction is uniformisable if the set-theoretic definition can be given in a form such that for each $A$ the corresponding $B$ is determined uniquely. A construction is natural if restriction from $B$ to its part $A$ always determines a map from the automorphism group of $B$ to that of $A$ which is a split surjective group homomorphism. We prove that there is no transitive model of ZFC (Zermelo-Fraenkel set theory with Choice) in which the uniformisable constructions are exactly the natural ones. We construct a transitive model of ZFC in which every uniformisable construction (with a restriction on the parameters in the formulas defining the construction) is 'weakly' natural. Corollaries are that the construction of algebraic closures of fields and the construction of divisible hulls of abelian groups have no uniformisations definable in ZFC without parameters.
\end{abstract}




\section{RESUMEN}

Consideramos una construcción algebraica como una aplicación conjuntista tomando estructuras $A$ a estructuras $B$ que tienen a $A$ como parte distinguida, de manera tal que cualquier isomorfismo de $A$ a $A^{\prime}$ se levanta a un isomorfismo de $B$ a $B^{\prime}$. En general la construcción define $B$ salvo isomorfismo sobre $A$. Una construcción es uniformizable si la definición conjuntista puede darse de forma tal que para cada $A$ el $B$ correspondiente está determinado únicamente. Una construcción es natural si la restricción de $B$ a su parte $A$ siempre determina una aplicación desde el grupo de automorfismos de $B$ al correspondiente de $A$ que es un homomorfismo de grupos sobreyectivo que escinde. Probamos que no existe un modelo transitivo de ZFC (teoría de conjuntos de Zermelo-Fraenkel con Axioma de Elección) en el cual las construcciones uniformizables sean exactamente las naturales. Construimos un modelo transitivo de ZFC en el cual toda construcción uniformizable (con una restricción en los parámetros de las fórmulas definiendo la construcción) es 'débilmente' natural. Como corolarios obtenemos que la construcción de clausuras algebraicas de cuerpos y la construcción de cápsulas divisibles de grupos abelianos no tienen uniformizaciones definibles en ZFC sin parámetros.

Keywords and Phrases: Naturality, uniformisability, transitive models, ZFC set theory

2010 AMS Mathematics Subject Classification: 08A35, 03E35 


\section{Introduction}

In two papers [4] and [6] we noted that in common practice many algebraic constructions are defined only 'up to isomorphism' rather than explicitly. We mentioned some questions raised by this fact, and we gave some partial answers. The present paper provides much fuller answers, though some questions remain open. Our main result, Theorem 5.1, implies at once that there is a transitive model of Zermelo-Fraenkel set theory with Choice (ZFC) in which every construction explicitly definable without parameters is 'weakly natural' (a weakening of the notion of a natural transformation). A corollary is that there are models of ZFC in which some well-known constructions, such as algebraic closure of fields, are not explicitly definable without parameters; some of these consequences were reported in [5]. We also show (Theorem 4.3) that there is no transitive model of ZFC in which the constructions explicitly definable (with parameters) are precisely the natural ones. The main questions left open are to extend Theorem 5.1 to constructions definable with parameters, and to determine whether Theorem 5.1 holds without the word 'weakly'.

Most of this work was done when the second author visited the first at Queen Mary, London University under SERC Visiting Fellowship grant GR/E9/639 in summer 1989, and later when the two authors took part in the Mathematical Logic year at the Mittag-Leffler Institute in Djursholm in September 2000. The first author had made a conjecture relating uniformisability to naturality. The second author proposed the approach of section 4 on the first occasion and the idea behind the proof of Theorem 5.1 on the second. Between 1975 and 2000 the authors (separately or together) had given some six or seven false proofs of versions of Theorem 5.1 or its negation. The authors thank Ian Hodkinson for his invaluable help (while research assistant to Hodges under SERC grant GR/D/33298) in unpicking some of the earlier false proofs. The first author also thanks the second author for his willingness to persist for several decades with these highly elusive problems.

\section{Constructions up to isomorphism}

To make this paper self-contained, we repeat or paraphrase some definitions from [6].

Definition 2.1. Let $M$ be a transitive model of $Z F C$. By a construction (in $M$ ) we mean a triple $\boldsymbol{C}=\left\langle\phi_{1}, \phi_{2}, \phi_{3}\right\rangle$ where

(1) $\phi_{1}(x), \phi_{2}(x)$ and $\phi_{3}(x)$ are formulas of the language of set theory, possibly with parameters from $M$;

(2) $\phi_{1}$ and $\phi_{2}$ respectively define first-order languages $L$ and $L^{-}$in $M$; every symbol of $L^{-}$is a symbol of $L$, and the symbols of $L \backslash L^{-}$include a 1-ary relation symbol $P$;

(3) the class $\left\{a: M \models \phi_{3}(a)\right\}$ is in $M$ a class of L-structures, called the graph of $\boldsymbol{C}$; 
(4) if $B$ is in the graph of $C$ then $P^{B}$, the set of elements of $B$ satisfying Px, forms the domain of an $L^{-}$-structure $B^{-}$inside $B$; thus if $Q$ is a relation symbol of $L^{-}$then $Q^{B^{-}}=Q^{B} \uparrow P^{B}$, and similarly for function symbols; the class of all structures $B^{-}$as $B$ ranges over the graph of $\boldsymbol{C}$ is called the domain of $\boldsymbol{C}$;

(5) the domain of $\boldsymbol{C}$ is closed under isomorphism; and if $A, B$ are in the graph of $\boldsymbol{C}$ then every isomorphism from $A^{-}$onto $B^{-}$extends to an isomorphism from $A$ onto $B$.

A typical example is the construction whose domain is the class of fields, and the structures $B$ in the graph are the algebraic closures of $B^{-}$, with $B^{-}$picked out by the relation symbol $P$. The algebraic closure of a field is determined only up to isomorphism over the field; in the terminology below, algebraic closures are 'representable' but not known to be 'uniformisable'. (What we called 'definable' in [6], and 'explicitly definable' in the introduction above, we now call 'uniformisable'; the new term agrees better with the common mathematical use of these words.)

Definition 2.2. (1) We say that the construction $\boldsymbol{C}$ is $X$-representable (in $M$ ) if $X$ is a set in $M$ and all the parameters of $\phi_{1}, \phi_{2}, \phi_{3}$ lie in $X$. We say that $\boldsymbol{C}$ is small if the domain of $\boldsymbol{C}$ (and hence also its graph) contains only a set of isomorphism types of structures.

(2) An important special case is where the domain of $\boldsymbol{C}$ contains exactly one isomorphism type of structure; in this case we say $\boldsymbol{C}$ is unitype.

The map $B^{-} \mapsto B$ on the domain of a construction $\mathbf{C}$ is in general not single-valued; but by clause (5) it is single-valued up to isomorphism over $B^{-}$.

Definition 2.3. (1) We say that $\boldsymbol{C}$ is uniformisable (in $M$ ) if its graph can be uniformised, i.e. there is a formula $\phi_{4}(x, y)$ of set theory (the uniformising formula) such that

for each $A$ in the domain of $C$ there is a unique $B$ such that $M \models \phi_{4}(A, B)$, and this $B$ is an L-structure in the graph of $C$ with $A=B^{-}$.

(2) We say that $\boldsymbol{C}$ is $X$-uniformisable (in $M$ ) if there is such a $\phi_{4}$ whose parameters lie in the set $X$.

\section{Splitting, naturality and weak naturality}

Definition 3.1. Let $\nu: G \rightarrow H$ be a surjective group homomorphism.

(i) A splitting of $\nu$ is a group homomorphism $s: H \rightarrow G$ such that $\nu$ s is the identity on $H$. We say that $\nu$ splits if it has a splitting.

(ii) By a weak splitting of $\nu$ we mean a set-theoretic map $s: H \rightarrow G$ such that 
(a) $\nu s$ is the identity on $H$;

(b) The composite map

$$
H \stackrel{s}{\longrightarrow} G \stackrel{\text { nat }}{\longrightarrow} G / \mathcal{Z}(G)
$$

is a homomorphism, where $\mathcal{Z}(G)$ is the centre of $G$ and nat is the natural homomorphism.

In particular every splitting is a weak splitting.

(iii) We say that $\nu$ weakly splits if it has a weak splitting.

Definition 3.2. Let $C$ be a construction. If $B$ is in the graph of $C$ and $A=B^{-}$, then by (4) in section 2, restriction from $B$ to $A$ induces a homomorphism $\nu: \operatorname{Aut}(B) \rightarrow \operatorname{Aut}(A)$, and by (5) this homomorphism is surjective. We say that $C$ is natural if for every such $B$ the homomorphism $\nu$ splits. We say that $\boldsymbol{C}$ is weakly natural if for every such $B$ the homomorphism $\nu$ weakly splits.

Note that if $\mathbf{C}$ is not (weakly) natural, then some structure $B$ in the graph of $\mathbf{C}$ witnesses this, so by restricting $\mathbf{C}$ to the isomorphism type of $B$ we get a unitype construction which is not (weakly) natural.

Example One. The construction of algebraic closures of fields is not weakly natural. The construction of divisible hulls of abelian groups is not weakly natural. Both these facts are proved in [5], using cohomology of finite abelian groups and (for the fields) some Galois theory. So they hold in any model of ZFC.

Example Two. There are constructions that are weakly natural but not natural. The simplest is as follows. The structures $B$ in the graph have six elements $a, b, c, d, e, f$ and the positive diagram

$$
P a, P b, R a c, R a e, R b d, R b f, S c d, S d e, S e f, S f c .
$$

The signature of $B$ consists of the relation symbols $P, R, S$, and the signature of $A=B^{-}$is empty. Then $\operatorname{Aut}(B)=\mathbb{Z} / 4 \mathbb{Z}, \operatorname{Aut}(A)=\mathbb{Z} / 2 \mathbb{Z}$ and $\nu: \operatorname{Aut}(B) \rightarrow \operatorname{Aut}(A)$ is the natural surjection. There is no splitting, because the automorphism of $A$ of order 2 lifts only to automorphisms of $B$ of order 4. But the construction is weakly natural because $\operatorname{Aut}(A)$ is abelian and hence is its own centre.

In [6] we conjectured that there are models of set theory in which each representable construction is uniformisable if and only if it is natural. Section 4 will show that no reasonable version of this conjecture is true. Sections 5 and 6 will show that there are models in which uniformisability implies weak naturality. Section 7 solves some of the problems raised in [4] and [6], and notes some connections with other things in the literature. 


\section{Uniformisability}

Definition 4.1. A structure $B$ is said to be rigid if it has no nontrivial automorphisms. We will say that a construction $\boldsymbol{C}$ is rigid-based if for every structure $B$ in the graph of $\boldsymbol{C}, B^{-}$has no nontrivial automorphisms.

A rigid-based construction is trivially natural.

Let $M$ be a transitive model of set theory. We will use a device that takes any construction $\mathbf{C}$ in $M$ to a construction $\mathbf{C}^{r}$, called its rigidification. The device exploits the fact that if $X$ is any nonempty set and $T C(X)$ is the transitive closure of $X$, then the structure $(T C(X), \epsilon)$ is rigid, thanks to the axiom of Foundation.

Suppose $B$ is in the graph of $\mathbf{C}$. Then without affecting any of the relevant isomorphisms, we can assume that none of the elements of $B$ outside $P^{B}$ lie in $T C\left(P^{B}\right)$. For example we can make a set-theoretic replacement of each element $b$ outside $P^{B}$ by the ordered pair $\left\langle b, T C\left(P^{B}\right)\right\rangle$.

To form $\mathbf{C}^{r}$, each structure $B^{-}$in the domain of $\mathbf{C}$ is replaced by a two-part structure $B^{r-}$, where the first part is $B^{-}$and the second part consists of the set $T C\left(P^{B}\right)$ with a membership relation $\varepsilon$ copying that in $M$. Now the structure $B^{r}$ is defined to be the amalgam of $B$ and $B^{r-}$, so that $B^{r-}$ is $\left(B^{r}\right)^{-}$. Then $\mathbf{C}^{r}$ is the closure of the class

$$
\left\{B^{r}: B \text { in the graph of } \mathbf{C}\right\}
$$

under isomorphism in $M$. It is clear that $\mathbf{C}^{r}$ and the map $B \mapsto B^{r}$ are definable in $M$ using no parameters beyond those in the formulas representing $\mathbf{C}$.

Lemma 4.2. If $\boldsymbol{C}$ is any construction, then $\boldsymbol{C}^{r}$ is rigid-based, natural and not small.

Proof. If $B^{-}$is in the domain of $\mathbf{C}$, then $B^{r-}$ is rigid because its set of elements is transitively closed; so $\mathbf{C}^{r}$ is rigid-based. Naturality follows at once. Since the domain of $\mathbf{C}$ is closed under isomorphism, the relevant transitive closures are arbitrarily large.

Theorem 4.3. There is no transitive model $M$ of ZFC in which both the following are true:

(a) Every rigid-based construction in $M$ is uniformisable.

(b) Every unitype uniformisable construction in $M$ is weakly natural.

In particular there is no transitive model of ZFC in which the natural constructions are exactly the uniformisable ones.

Proof. Suppose $M$ is a counterexample. By Example One in section 3 there are some nonweakly-natural constructions in $M$. So by restricting to a single isomorphism type we can find a 
unitype non-weakly-natural construction $\mathbf{C}$ in $M$. Then $\mathbf{C}^{r}$ is rigid-based and hence uniformisable by assumption. But we can use the uniformising formula of $\mathbf{C}^{r}$ to uniformise $\mathbf{C}$ with the same parameters. So by the assumption on $M$ again, $\mathbf{C}$ is weakly natural; contradiction.

The next result gives some finer information about small constructions.

Theorem 4.4. Let $M$ be a transitive model of ZFC, $Y$ a set in $M$ and $\bar{c}$ a well-ordering of $Y$ in M. Assume:

In $M$, if $X$ is any set, then every unitype $X$-representable rigid-based construction is $X \cup Y$-uniformisable.

Then

In $M$, every small $\emptyset$-representable construction is $\{\bar{c}\}$-uniformisable,

and hence there are unitype $\{\bar{c}\}$-uniformisable constructions that are not weakly natural.

Proof. Let $\gamma$ be the length of $\bar{c}$. Write $\bar{v}$ for the sequence of variables $\left(v_{i}: i<\gamma\right)$. In $M$ we can well-order (definably, with no parameters) the class of pairs $\langle j, \psi\rangle$ where $j$ is an ordinal and $\psi(x, y, z, \bar{v})$ is a formula of set theory. We write $H_{j}$ for the set of sets hereditarily of cardinality less than $\aleph_{j}$ in $M$.

Let $\mathbf{C}$ be a small $\emptyset$-representable construction in $M$. Then $\mathbf{C}^{r}$ is an $\emptyset$-representable rigid-based construction. It is not small; but if $B$ is any structure in the graph of $\mathbf{C}$, let $\mathbf{C}_{B}$ be the construction got from $\mathbf{C}^{r}$ by restricting the graph to structures isomorphic to $B^{r}$. Then $\mathbf{C}_{B}$ is a unitype and $\{B\}$-representable rigid-based construction, so by assumption it is $\{B\} \cup Y$-uniformisable, say by a formula $\psi_{B}(-,-, B, \bar{c})$ where $B, \bar{c}$ are the parameters.

By the reflection principle in $M$ there is an ordinal $j$ such that

$$
M \models \exists C\left(C \in \mathbf{C}_{B} \wedge C^{-}=B^{r-} \wedge C \text { is the unique set such that " } H_{j} \models \psi_{B}\left(B^{r-}, C, B, \bar{c}\right)\right. \text { "). }
$$

Hence in $M$ there is a first pair $\left\langle j_{B}, \psi_{B}\right\rangle$, definable from $B$, such that

$$
M \models \exists C\left(C \in \mathbf{C}_{B} \wedge C^{-}=B^{r-} \wedge C \text { is the unique set such that " } H_{j_{B}} \models \psi_{B}\left(B^{r-}, C, B, \bar{c}\right)\right. \text { "). }
$$

Since all of this is uniform in $B$, it follows that the construction $\mathbf{C}$ is $\{\bar{c}\}$-uniformisable in $M$ by the formula $\phi(x, y, \bar{c})$ which says

$$
y=C \mid L \text { where } H_{j_{x}} \models \psi_{x}\left(x^{r-}, C, x, \bar{c}\right) .
$$

The last clause of the theorem follows by choosing $\mathbf{C}$ suitably, for example using Example One of section 3 . 


\section{The set theory}

Theorem 5.1. Let $M$ be a countable transitive model of $Z F C$ and GCH, and $\lambda$ a transfinite cardinal in $M$. Then there is a forcing extension $N$ of $M$ with the following property. If $\boldsymbol{C}$ is a uniformisable unitype construction defined in $N$ with parameters in $M$, whose graph contains a structure $B$ in $M$ with $B$ and $\operatorname{Aut}(B)$ both of cardinality $\leqslant \lambda$, then $C$ is weakly natural in $N$.

The proof of this theorem will occupy this and the next section. The idea is to consider any unitype construction $\mathbf{C}$ whose parameters lie in $M$, and introduce a very homogeneous generic structure $B^{\star}$ into the graph of $\mathbf{C}$. The homogeneity of $B^{\star}$ will make it impossible to uniformise without some form of naturality. This is a novel argument. At present we can apply it simultaneously for all unitype constructions satisfying the stated restriction to a fixed $\lambda$. We expect that a similar proof by class forcing will eliminate this restriction, but this is delayed.

Our notation for forcing mainly follows Jech [7]. We define $\mathbb{P}$ to be the notion of forcing in $M$ that consists of all partial maps from $\lambda^{++} \times \lambda^{++} \times \lambda^{++}$to 2 which have domain of cardinality at most $\lambda$. We abbreviate $\lambda^{++} \times \lambda^{++} \times \lambda^{++}$to $\left(\lambda^{++}\right)^{3}$.

Lemma 5.2. The notion of forcing $\mathbb{P}$ is $\lambda^{+}$-closed and satisfies the $\lambda^{++}$-chain condition.

For definiteness we take $M^{\mathbb{P}}$, the class of $\mathbb{P}$-names, to be the smallest class of elements of $M$ such that if $X$ is any subset of $M^{\mathbb{P}}$ and for each $y \in X, I_{y}$ is a non-empty antichain in $\mathbb{P}$, then $\left\{(p, y): y \in X, p \in I_{y}\right\}$ is a $\mathbb{P}$-name in $M^{\mathbb{P}}$; the domain of this $\mathbb{P}$-name is $X$. Then for every $\mathbb{P}$-generic $G$ the interpretation of the name $\dot{x}=\left\{(p, y): y \in X, p \in I_{y}\right\}$ is the set $\dot{x}[G]=\{y[G]: \exists p \in G,(p, y) \in \dot{x}\}$. We write $\dot{x}$ for $\mathbb{P}$-names, and $\check{x}$ for the canonical $\mathbb{P}$-name of the element $x \in M$.

We take a $\mathbb{P}$-generic set $G$ over $M$ and we put $N=M[G]$. We will prove Theorem 5.1 for this $N$. In $M$ we fix a unitype construction $\mathbf{C}$, a structure $B$ in the graph of $\mathbf{C}$, and a uniformising formula $\phi(x, y)$. We write $A$ for $B^{-}$.

Definition 5.3. In $M$ we define two homomorphisms, I from the group of permutations of $\left(\lambda^{++}\right)^{3}$ to the group of automorphisms of $\mathbb{P}$ as ordered set; and $J$ from the group of automorphisms of $\mathbb{P}$ to the group of permutations of $M^{\mathbb{P}}$. Thus:

(a) Let $\alpha$ be a permutation of $\left(\lambda^{++}\right)^{3}$ and $p \in \mathbb{P}$. Then we define $\alpha^{I}(p)$ by

$$
\left(\alpha^{I}(p)\right)(\alpha(i, j, k))=p(i, j, k) \text { for all } i, j, k<\lambda^{++} .
$$


(b) Let $\gamma$ be an automorphism of $\mathbb{P}$. Then $\gamma^{J}$ is defined on $M^{\mathbb{P}}$ by induction on rank:

$$
\gamma^{J} \dot{x}=\left\{\left(\gamma p, \gamma^{J} \dot{y}\right):(p, \dot{y}) \in \dot{x}\right\} .
$$

The maps $I$ and $J$ are clearly homomorphisms.

Lemma 5.4. Let $\gamma$ be an automorphism of $\mathbb{P}$ which is in $M$. Then:

(a) If $G$ is a $\mathbb{P}$-generic set over $M$, then $\gamma G$ is $\mathbb{P}$-generic over $M$, and for every $\mathbb{P}$-name $\dot{x}$ we have

$$
\left(\gamma^{J} \dot{x}\right)[\gamma G]=\dot{x}[G]
$$

(where $\gamma G=\{\gamma p: p \in G\})$.

(b) If $\dot{x}$ is a $\mathbb{P}$-name then $(\alpha) \Rightarrow(\beta)$, where we write

$(\alpha)$ : for every pair $(p, \dot{y}),(p, \dot{y}) \in \dot{x}$ if and only if $\left(\gamma p, \gamma^{J} \dot{y}\right) \in \dot{x}$.

$(\beta): \gamma^{J}(\dot{x})=\dot{x}$.

Proof. . For (a), by induction on the rank of $\dot{x}$,

$$
\begin{aligned}
\dot{x}[G] & =\{\dot{y}[G]: \exists p \in G,(p, \dot{y}) \in \dot{x}\} \\
& =\left\{\gamma^{J} \dot{y}[\gamma G]: \exists \gamma p \in \gamma G,\left(\gamma p, \gamma^{J} \dot{y}\right) \in \gamma^{J} \dot{x}\right\} \\
& =\left\{\dot{z}[\gamma G]: \exists q \in \gamma G,(q, \dot{z}) \in \gamma^{J} \dot{x}\right\} \\
& =\left(\gamma^{J} \dot{x}\right)[\gamma G] .
\end{aligned}
$$

Part (b) is immediate from the definition of $\gamma^{J}$.

Since $G$ is $\mathbb{P}$-generic, $\bigcup G$ is a total map from $\left(\lambda^{++}\right)^{3}$ to 2 . For each $i<\lambda^{++}$and $j<\lambda^{++}$, we define $a_{i j}=\left\{k<\lambda^{++}: \bigcup G(i, j, k)=1\right\}$ and $a_{i}^{\prime}=\left\{a_{i j}: j<\lambda^{+}\right\}$, so that $a_{i}^{\prime}$ is a set of $\lambda^{++}$ independently generic subsets of $\lambda^{++}$. If $a$ and $b$ are (in $N$ ) sets of subsets of $\lambda^{++}$, we put $a \equiv b$ iff the symmetric difference of $a$ and $b$ has cardinality $\leqslant \lambda$. We write $a_{i}$ for the equivalence class $\left(a_{i}^{\prime}\right)^{\equiv}$. The $\mathbb{P}$-names $\dot{a}_{i j}, \dot{a}_{i}^{\prime}, \dot{a}_{i}$ can be chosen in $M^{\mathbb{P}}$ independently of the choice of $G$.

Consider again the structures $A$ and $B$ in $M$. Without loss we can suppose that $\operatorname{dom}(A)$ is an initial segment of $\lambda$. In $M[G]$ there is a map $e$ which takes each element $i$ of $A$ to the corresponding set $a_{i}=\dot{a}_{i}[G]$; by means of $e$ we can define a copy $A^{\star}$ of $A$ whose elements are the sets $a_{i}(i \in \operatorname{dom}(A))$.

Lemma 5.5. The $\mathbb{P}$-names $\dot{A}^{\star}, \dot{e}$ can be chosen to be independent of the choice of $G$. Also we can take the boolean names $\dot{a}_{i j}$ and $\dot{a}_{i}^{\prime}$ to be

$$
\begin{aligned}
\dot{a}_{i j} & =\left\{(((i, j, k) \mapsto 1), \check{k}):(i, j, k) \in\left(\lambda^{++}\right)^{3}\right\} \\
\dot{a}_{i}^{\prime} & =\left\{\left(1, \dot{a}_{i j}\right): j<\lambda^{++}\right\} .
\end{aligned}
$$


A notion of forcing $\mathbb{Q}$ in $M$ is said to be homogeneous if for any two conditions $p, q \in \mathbb{P}$ there is an automorphism $\alpha$ of $\mathbb{Q}$ in $M$ such that $p$ and $\alpha q$ are compatible.

Lemma 5.6. $\mathbb{P}$ is homogeneous.

By this lemma and the fact that $A, B$ and the parameters of the uniformising formula $\phi$ lie in $M$, the statement " $\phi$ uniformises a construction on the class of structures isomorphic to $A$, which takes $A$ to $B$ " is true in $N$ independently of the choice of $G$. In particular there are $\mathbb{P}$-names $\dot{B}^{\star}, \dot{\varepsilon}$ such that

$\| \dot{B}$ is the unique structure such that $\phi\left(\dot{A}^{\star}, \dot{B}^{*}\right)$ holds,

$\dot{e}: \check{A} \rightarrow \dot{A}^{*}$ is the isomorphism such that $\dot{e}(\check{\imath})=\dot{a}_{i}$ for each $i \in \operatorname{dom}(\check{A})$, and $\dot{\varepsilon}: \check{B} \rightarrow \dot{B}^{*}$ is an isomorphism which extends $\dot{e} \|_{\mathbb{P}}=1$.

Lemma 5.7. Let $G$ be $\mathbb{P}$-generic over $M$. Then:

(a) $\operatorname{Aut}(A)^{M}=\operatorname{Aut}(A)^{M[G]}$.

(b) $\operatorname{Aut}(B)^{M}=\operatorname{Aut}(B)^{M[G]}$.

(c) The set of maps from $\operatorname{Aut}(A)$ to $\operatorname{Aut}(B)$ is the same in $M$ as it is in $M[G]$.

Proof. . $\mathbb{P}$ is $\lambda^{+}$-closed by Lemma 5.2. Hence no new permutations of $A$ or $B$ are added since $|A| \leq|B| \leq \lambda$ in $M$; this proves (a), (b). Likewise (c) holds since $|\operatorname{Aut}(A)| \leq|\operatorname{Aut}(B)| \leq \lambda$ in $M$.

We regard $\operatorname{Aut}(A)$ as a permutation group on $\lambda^{++}$by letting it fix all the elements of $\lambda^{++}$ which are not in $\operatorname{dom}(A)$.

We write $\Pi$ for the cartesian product $\prod_{\lambda^{++}} \operatorname{Aut}(A)$ of $\lambda^{++}$copies of the $\operatorname{group} \operatorname{Aut}(A)$, in the sense of $M$. Then each element $\alpha$ of $\Pi$ can be regarded as a map $\alpha: \lambda^{++} \rightarrow \operatorname{Aut}(A)$ in $M$. We write $\mathcal{N}$ for the subgroup of $\Pi$ consisting of those $\alpha$ such that for some finite sequence of ordinals

$$
0=i_{0}<i_{1}<\ldots<i_{n}<i_{n+1}=\lambda^{++}
$$

the map $\alpha$ is constant on each interval $\left[i_{k}, i_{k+1}\right)(0 \leqslant k \leqslant n)$. The elements of $\mathcal{N}$ will be called neat maps. We write $\pi$ for the map from $\mathcal{N}$ to $\operatorname{Aut}(A)$ which takes each neat map to its eventual value. We write $\mathcal{N}^{-}$for the set of all neat maps $\alpha$ with $\pi(\alpha)=1$. For each ordinal $i<\lambda^{++}$we write $\mathcal{N}_{i}$ for the set of neat maps $\alpha$ such that $\alpha(j)=1$ for all $j<i$. We write $\mathcal{N}_{i}^{-}$for $\mathcal{N}^{-} \cap \mathcal{N}_{i}$. 
Lemma 5.8. As a subset of the group $\Pi, \mathcal{N}$ forms a group with subgroups $\mathcal{N}^{-}, \mathcal{N}_{i}\left(i<\lambda^{++}\right)$. The map $\pi: \mathcal{N} \rightarrow \operatorname{Aut}(A)$ is a surjective group homomorphism.

Proof. . From the definitions.

The neat map $\alpha \in \Pi$ determines a permutation $\alpha^{K}$ of the set $\left(\lambda^{++}\right)^{3}$ by

$$
\alpha^{K}(i, j, k)=(\alpha(j)(i), j, k) .
$$

Hence $\alpha$ induces an automorphism $\alpha^{K I J}$ of $M^{\mathbb{P}}$.

Lemma 5.9. Suppose $\alpha: \lambda^{++} \rightarrow \operatorname{Aut}(A)$ is neat. Then $\alpha^{K I J}$ setwise fixes the set $\left\{\dot{a}_{i}: i \in\right.$ $\operatorname{dom}(A)\}$ of canonical names of the elements of $\dot{A}^{*}[G]$, and it acts on this set in the way induced by $\pi(\alpha)$ and the map $i \mapsto \dot{a}_{i}$. Thus $\alpha^{K I J}\left(\dot{a}_{i}\right)=\dot{a}_{\pi(\alpha)(i)}$.

Proof. . We use the boolean names in Lemma 5.5. For $\dot{a}_{i j}$,

$$
\begin{aligned}
\alpha^{K I J} \dot{a}_{i j} & =\left\{\left(\alpha^{K I}((i, j, k) \mapsto 1), \alpha^{K I J}(\check{k})\right):(i, j, k) \in\left(\lambda^{++}\right)^{3}\right\} \\
& =\left\{\left(\left(\alpha^{K}(i, j, k) \mapsto 1\right), \check{k}\right):(i, j, k) \in\left(\lambda^{++}\right)^{3}\right\} \\
& \left.=\{(\alpha(j)(i), j, k) \mapsto 1), \check{k}):(i, j, k) \in\left(\lambda^{++}\right)^{3}\right\} \\
& =\dot{a}_{\alpha(j) i, j}
\end{aligned}
$$

Then for $\dot{a}_{i}^{\prime}$,

$$
\begin{aligned}
\alpha^{K I J} \dot{a}_{i}^{\prime} & =\left\{\left(\alpha^{K I}\left(1, \dot{a}_{i j}\right): j<\lambda^{++}\right\}\right. \\
& =\left\{\left(1, \dot{a}_{\alpha(j) i, j}\right): j<\lambda^{++}\right\} .
\end{aligned}
$$

We claim that with boolean value $1,\left\{\left(1, \dot{a}_{\alpha(j) i, j}\right): j<\lambda^{++}\right\} \equiv \dot{a}_{\pi \alpha(i)}^{\prime}$. For this, first note that

$$
\dot{a}_{\pi \alpha(i)}^{\prime}=\left\{\left(1, \dot{a}_{\pi(\alpha) i, j}\right): j<\lambda^{++}\right\} .
$$

Since $\alpha$ is neat, there is $j_{0}<\lambda^{++}$such that $\alpha(j)=\pi \alpha$ whenever $j \geqslant j_{0}$. So for any generic $G$, $\left\{\left(1, \dot{a}_{\alpha(j) i, j}\right): j<\lambda^{++}\right\}[G]$ and $\dot{a}_{\pi \alpha(i)}^{\prime}[G]$ differ in at most $\left|j_{0}\right|$ elements. The lemma follows.

Lemma 5.10. For each element $i$ of $A$ and each neat map $\alpha, \dot{a}_{\pi(\alpha)(i)}[\alpha G]=\dot{a}_{i}[G]$. In particular $\dot{A}^{\star}[\alpha G]=\dot{A}^{\star}[G]$.

Proof. By Lemma $5.9, \dot{a}_{\pi(\alpha)(i)}[\alpha G]=\left(\alpha \dot{a}_{i}\right)[\alpha G]$. Then by Lemma 5.4 and the fact that $\alpha \dot{a}_{i}$ lies in $M^{\mathbb{P}}$,

$$
\left(\alpha \dot{a}_{i}\right)[\alpha G]=\dot{a}_{i}[G]
$$

This shows that $\dot{A}^{\star}[\alpha G]=\dot{A}^{\star}[G]$. 
We write $\dot{\varepsilon}^{-1}$ for a $\mathbb{P}$-name such that $\dot{\varepsilon}^{-1}[G]=(\dot{\varepsilon}[G])^{-1}$ for all generic $G$.

Lemma 5.11. Suppose $\alpha$ is a neat map and $G$ is $\mathbb{P}$-generic over $M$. Then $\dot{B}^{*}\left[\alpha^{-1} G\right]=\dot{B}^{*}[G]$, and the map $\left(\dot{\varepsilon}^{-1} \circ \alpha \dot{\varepsilon}\right)[G]$ is an automorphism of $B$ which extends $\pi(\alpha)$.

Proof. Since $M\left[\alpha^{-1} G\right]=M[G]$ and $\dot{A}^{*}\left[\alpha^{-1} G\right]=\dot{A}^{*}[G]$, statement (5.1) (before Lemma 5.7) tells us that $\dot{e}\left[\alpha^{-1} G\right](i)=\dot{a}_{i}\left[\alpha^{-1} G\right]$ for each $i \in \operatorname{dom}(A)$, and that $\dot{B}^{*}\left[\alpha^{-1} G\right]=\dot{B}^{*}[G]$ and $\dot{\varepsilon}[G]^{-1} \circ \dot{\varepsilon}\left[\alpha^{-1} G\right]$ extends $\dot{e}[G]^{-1} \circ \dot{e}\left[\alpha^{-1} G\right]$. Now using Lemma 5.10,

$$
\begin{gathered}
\dot{e}[G]^{-1} \circ \dot{e}\left[\alpha^{-1} G\right](i)=\dot{e}[G]^{-1}\left(\dot{a}_{i}\left[\alpha^{-1} G\right]\right) \\
=\dot{e}[G]^{-1}\left(\dot{a}_{\pi(\alpha)(i)}[G]\right)=\pi(\alpha)(i) .
\end{gathered}
$$

Lemma 5.12. For every neat map $\alpha$ and all $p \in \mathbb{P}$ there are $p^{\prime} \leqslant p$ and $g \in$ Aut $B$ extending $\pi(\alpha)$, such that

$$
p^{\prime} \vdash_{\mathbb{P}} \dot{\varepsilon}^{-1} \circ \alpha(\dot{\varepsilon})=\check{g} .
$$

Proof. Let $f$ be $\pi(\alpha)$. By Lemma 5.11 we have

$$
\begin{aligned}
1 & =\| \dot{\varepsilon}^{-1} \circ \alpha \dot{\varepsilon} \text { is an automorphism of } B \text { extending } \check{f} \|_{\mathbb{P}} \\
& =\sum_{g}\left\|\dot{\varepsilon}^{-1} \circ \alpha \dot{\varepsilon}=\check{g}\right\|_{\mathbb{P}}
\end{aligned}
$$

where $g$ ranges over the automorphisms of $B$ that extend $f$.

Definition 5.13. (a) For each $p \in \mathbb{P}$ and each $i<\lambda^{++}$, define $t_{p, i}$ to be the set of all pairs $(f, g)$, with $f \in \operatorname{Aut}(A)$ and $g \in \operatorname{Aut}(B)$, such that for some $\alpha \in \mathcal{N}_{i}, \pi(\alpha)=f$ and

$$
p \vdash_{\mathbb{P}} \dot{\varepsilon}^{-1} \circ \alpha \dot{\varepsilon}=\check{g} .
$$

(b) Clearly if $p^{\prime} \leqslant p$ then $t_{p^{\prime}, i} \supseteq t_{p, i}$. The number of possible values for $f$ and $g$ is $\leqslant \lambda$ by choice of $\lambda$, and $\mathbb{P}$ is $\lambda^{+}$-closed; so there is $p_{i}$ such that for all $p^{\prime} \leqslant p_{i}$,

$$
t_{p^{\prime}, i}=t_{p_{i}, i}
$$

We fix a choice of $p_{i}$ for each $i$, and we write $t_{i}$ for the resulting value $t_{p_{i}, i}$.

(c) For each $i$ and each $(f, g)$ in $t_{i}$ we choose $\alpha$ in $\mathcal{N}_{i}$ with $\pi(\alpha)=f$ so that

$$
p_{i} \Vdash_{\mathbb{P}} \dot{\varepsilon}^{-1} \circ \alpha \dot{\varepsilon}=\check{g} .
$$

We write $\alpha_{f, g}^{i}$ for this $\alpha$. 
Lemma 5.14. For each $i<\lambda^{++}, t_{i}$ is a subset of $\operatorname{Aut}(A) \times \operatorname{Aut}(B)$ such that

(a) for each $(f, g)$ in $t_{i}, g \mid A=f$;

(b) for each $f$ in $\operatorname{Aut}(A)$ there is $g$ with $(f, g)$ in $t_{i}$.

$\left(\right.$ So $_{i}(-,-)$ is a first attempt at a lifting of the restriction map from $\operatorname{Aut}(B)$ to $\operatorname{Aut}(A)$.)

Proof. By Lemma 5.12 and the surjectivity of $\pi$.

Lemma 5.15. There is a stationary subset $S$ of $\lambda^{++}$such that:

(a) for each $i \in S$ and $j<i$, the domain of $p_{j}$ is a subset of $i \times i \times i$;

(b) for each $i \in S$ and $j<i$, every map $\alpha_{f, g}^{j}: \lambda^{++} \rightarrow \operatorname{Aut}(A)$ is constant on $\left[i, \lambda^{++}\right)$;

(c) for all $i, j \in S, t_{i}=t_{j}$;

(d) there is a condition $p^{\star} \in \mathbb{P}$ such that for all $i \in S, p_{i} \uparrow(i \times i \times i)=p^{\star}$.

Proof. First, there is a club $C \subseteq \lambda^{++}$on which (a) and (b) hold. Let $S_{\eta}$ be $\left\{\delta<\lambda^{++}: \operatorname{cf}(\delta)=\lambda^{+}\right\}$. Clearly $S_{\nu}=S_{\eta} \cap C$ is stationary; and for each $i \in S_{\nu}, p_{i} \uparrow(i \times i \times i)$ has domain $\subseteq j \times j \times j$ for some $j=j_{i}<i$. Then by Födor's lemma there is a stationary subset $S$ of $S_{\nu}$ on which (c) and (d) hold.

\section{The weak lifting}

Continuing Section 5, we use the notation $S, p^{\star}$ from Lemma 5.15. We write $t$ for the constant value of $t_{i}(i \in S)$ from clause (c) of Lemma 5.15, and $t^{-}$for the set of all $g$ such that $(1, g) \in t$. We write $\nu: \operatorname{Aut}(B) \rightarrow \operatorname{Aut}(A)$ for the restriction map. If $X$ is a subset of $\operatorname{Aut}(B)$, we write $\langle X\rangle$ for the subgroup of $\operatorname{Aut}(B)$ generated by $X$.

Lemma 6.1. The relation $t$ is a subset of $\operatorname{Aut}(A) \times \operatorname{Aut}(B)$ that projects onto $\operatorname{Aut}(A)$, and if $(f, g)$ is in $t$ then $\nu(g)=f$.

Proof. This repeats Lemma 5.14 (a) and (b).

Lemma 6.2. If $\left(f_{1}, g_{1}\right)$ and $\left(f_{2}, g_{2}\right)$ are both in $t$ then $\left(f_{1} f_{2}, g_{1} g_{2}\right)$ is in $t$. 
Proof. Take any $i, j \in S$ with $i<j$. Put $\alpha_{1}=\alpha_{f_{1}, g_{1}}^{j}, \alpha_{2}=\alpha_{f_{2}, g_{2}}^{i}$ and $\alpha_{3}=\alpha_{1} \alpha_{2}$. Note that $\alpha_{1} \alpha_{2}$ is in $\mathcal{N}_{i}$ since $i<j$.

Trivially we have

$$
p_{j} \Vdash \dot{\varepsilon}^{-1} \circ \alpha_{3}(\dot{\varepsilon})=\dot{\varepsilon}^{-1} \circ \alpha_{1}(\dot{\varepsilon}) \circ\left(\alpha_{1}(\dot{\varepsilon})\right)^{-1} \circ \alpha_{3}(\dot{\varepsilon})
$$

and by assumption

$$
p_{j} \Vdash \dot{\varepsilon}^{-1} \circ \alpha_{1}(\dot{\varepsilon})=\check{g_{1}}
$$

So

$$
p_{j} \Vdash \dot{\varepsilon}^{-1} \circ \alpha_{3}(\dot{\varepsilon})=\check{g_{1}} \circ\left(\alpha_{1}(\dot{\varepsilon})\right)^{-1} \circ \alpha_{1}\left(\alpha_{2} \dot{\varepsilon}\right) .
$$

Also by assumption

$$
p_{i} \Vdash \dot{\varepsilon}^{-1} \circ \alpha_{2}(\dot{\varepsilon})=\check{g_{2}} .
$$

Acting on this last formula by $\alpha_{1}$ gives

$$
\alpha_{1} p_{i} \Vdash \alpha_{1} \dot{\varepsilon}^{-1} \circ \alpha_{1} \alpha_{2} \dot{\varepsilon}=\alpha_{1} \check{g_{2}} .
$$

Now $\alpha_{1} \check{g_{2}}=\check{g_{2}}$. Also $\alpha_{1} p_{i}=p_{i}$ since the support of $p_{i}$ lies entirely below $j$ (by Lemma $5.15(\mathrm{a})$ ), and $\alpha_{1}=\alpha_{f_{1}, g_{1}}^{j}$ is the identity in this region since it lies in $\mathcal{N}_{j}$. So we have shown that

$$
p_{i} \Vdash \alpha_{1} \dot{\varepsilon}^{-1} \circ \alpha_{1} \alpha_{2} \dot{\varepsilon}=\check{g_{2}} .
$$

Now we note that $p_{i} \cup p_{j}$ is a condition in $\mathbb{P}$, by (a), (d) of Lemma 5.15. Hence we have that

$$
p_{i} \cup p_{j} \Vdash \dot{\varepsilon}^{-1} \circ \alpha_{3} \dot{\varepsilon}=\check{g_{1}} \check{g_{2}} .
$$

Since $\alpha_{3}$ is in $\mathcal{N}_{i}$, this shows that

$$
\left(f_{1} f_{2}, g_{1} g_{2}\right) \in t_{p_{i} \cup p_{j}, i} .
$$

Then by the maximality property of $p_{i}$,

$$
\left(f_{1} f_{2}, g_{1} g_{2}\right) \in t_{p_{i}, i}
$$

so that $\left(f_{1} f_{2}, g_{1} g_{2}\right)$ is in $t$.

Corollary 6.3. If $\left(f, g_{1}\right)$ and $\left(f, g_{2}\right)$ are in $t$ then $g_{1} g_{2}^{-1}$ is in $\left\langle t^{-}\right\rangle$. 
Proof. By Lemma 6.1 there is some $g^{\prime} \in \operatorname{Aut}(B)$ such that $\left(f^{-1}, g^{\prime}\right)$ is in $t$. Then by Lemma 6.2, $\left(1, g_{1} g^{\prime}\right)$ and $\left(1, g_{2} g^{\prime}\right)$ are in $t$ and so $g_{1} g^{\prime}, g_{2} g^{\prime}$ are in $t^{-}$. Hence the element

$$
g_{1} g_{2}^{-1}=\left(g_{1} g^{\prime}\right)\left(g_{2} g^{\prime}\right)^{-1}
$$

lies in $\left\langle t^{-}\right\rangle$.

Lemma 6.4. Every element of $t^{-}$is central in $\operatorname{Aut}(B)$.

Proof. Suppose $g_{2} \in t^{-}$, so that $\left(1, g_{2}\right) \in t$. Consider $\left(f_{1}, g_{2}\right) \in t$, and apply the notation of the proof of Lemma 6.2 with $f_{2}=1$. In that notation, $\alpha_{1}$ is the identity below $j$ and $\alpha_{2}$ is the identity below $i$ (since $i, j \in S$ ). But also $g_{2}$ lies in $t^{-}$, so $\alpha_{2}$ is the identity on $\left[j, \lambda^{+}\right.$). In particular $\alpha_{1}$ commutes with $\alpha_{2}$.

As in the proof of Lemma 6.2 we have

$$
p_{i} \Vdash \dot{\varepsilon}^{-1} \circ \alpha_{3} \dot{\varepsilon}=\dot{\varepsilon}^{-1} \circ \alpha_{2} \dot{\varepsilon} \circ \alpha_{2} \dot{\varepsilon}^{-1} \circ \alpha_{3} \dot{\varepsilon} .
$$

As before, we have that

$$
p_{i} \Vdash \dot{\varepsilon}^{-1} \circ \alpha_{2} \dot{\varepsilon}=\check{g_{2}}
$$

and

$$
\alpha_{2} p_{j} \Vdash \alpha_{2} \dot{\varepsilon}^{-1} \circ \alpha_{2} \alpha_{1} \dot{\varepsilon}=\alpha_{2} \check{g_{1}} .
$$

Now the support of $p_{j}$ lies below $i$ or within $\left[j, \lambda^{+}\right) \times \operatorname{dom} A$, and $\alpha_{2}$ is the identity in both these regions, and so $\alpha_{2}\left(p_{j}\right)=p_{j}$. Thus, since $\alpha_{1}$ commutes with $\alpha_{2}$,

$$
p_{j} \Vdash \alpha_{2} \dot{\varepsilon}^{-1} \circ \alpha_{3} \dot{\varepsilon}=\check{g_{1}} \text {. }
$$

So as before,

$$
p_{i} \cup p_{j} \Vdash \dot{\varepsilon}^{-1} \circ \alpha_{3} \dot{\varepsilon}=\check{g_{2}} \check{g_{1}} .
$$

Recalling that in the proof of Lemma 6.2 we showed that

$$
p_{i} \cup p_{j} \Vdash \dot{\varepsilon}^{-1} \circ \alpha_{3}(\dot{\varepsilon})=\check{g_{1}} \check{g_{2}},
$$

we deduce that

$$
p_{i} \cup p_{j} \Vdash \check{g_{1}} \check{g_{2}}=\check{g_{2}} \check{g_{1}} .
$$

But the equation $g_{1} g_{2}=g_{2} g_{1}$ is about the ground model, and hence it is true. 
Now in $M$ choose a map $s: \operatorname{Aut}(A) \rightarrow \operatorname{Aut}(B)$ so that for each $f \in \operatorname{Aut}(A), s(f)$ is some $g$ with $(f, g) \in t$. This is possible by Lemma 6.1.

Lemma 6.5. In $M$ the map $s$ is a weak splitting of $\nu: \operatorname{Aut}(B) \rightarrow \operatorname{Aut}(A)$.

Proof. Trivially $\nu s$ is the identity on $\operatorname{Aut}(A)$. Write $s^{\prime}: \operatorname{Aut}(A) \rightarrow \mathcal{Z}(\operatorname{Aut}(B))$ for the composite of $s$ and nat: $\operatorname{Aut}(B) \rightarrow \mathcal{Z}(\operatorname{Aut}(B))$. We show that $s^{\prime}$ is a homomorphism as follows. Suppose $f_{1} f_{2}=f_{3}$ in $\operatorname{Aut}(A)$. Put $g_{i}=s\left(f_{i}\right)$ for each $i(1 \leqslant i \leqslant 3)$. Then by Lemma $6.2,\left(f_{3}, g_{1} g_{2}\right)$ is in $t$, so by Corollary 6.3 and Lemma $6.4, g_{1} g_{2} g_{3}^{-1}$ is in $\left\langle t^{-}\right\rangle \subseteq \mathcal{Z}(\operatorname{Aut}(B))$. Then

$$
\begin{aligned}
s^{\prime}\left(f_{1}\right) \sigma^{\prime}\left(f_{2}\right) & =g_{1} \mathcal{Z}(\operatorname{Aut}(B)) \cdot g_{2} \mathcal{Z}(\operatorname{Aut}(B)) \\
& =g_{1} g_{2} \cdot \mathcal{Z}(\operatorname{Aut}(B)) \\
& =g_{3} \mathcal{Z}(\operatorname{Aut}(B))=s^{\prime}\left(f_{3}\right)
\end{aligned}
$$

as required.

This completes the proof of Theorem 5.1.

\section{Answers to questions}

The results above answer most of the problems stated in [6]. In that paper we showed:

Theorem $\mathbf{3}$ of [6] If $\mathbf{C}$ is a small natural construction in a model of ZFC, then $\mathbf{C}$ is uniformisable with parameters.

We asked (Problem A) whether it is possible to remove the restriction that $\mathbf{C}$ is small. The answer is No:

Theorem 7.1. There is a transitive model of ZFC in which some $\emptyset$-representable construction is natural but not uniformisable (even with parameters).

Proof. Let $N$ be the model of Theorem 5.1. Let $\mathbf{C}$ be some construction $\emptyset$-representable in $N$ which is not weakly natural (cf. Example One in section 3). Then by Theorem 5.1, C is not uniformisable. The rigidifying construction $\mathbf{C}^{r}$ of section 3 is $\emptyset$-representable, natural and not uniformisable.

Problem B asked whether in Theorem 3 of [6] the formula defining $\mathbf{C}$ can be chosen so that it has only the same parameters as the formulas chosen to represent $\mathbf{C}$. The answer is No:

Theorem 7.2. There is a transitive model $N$ of ZFC with the following property: 
For every set $Y$ there are a set $X$ and a unitype rigid-based (hence small natural) $X$-representable construction that is not $X \cup Y$-uniformisable.

Proof. Take $N$ to be the model given by Theorem 5.1. Let $Y$ be any set in $N$. If $N$ and $Y$ are not as stated above, then for every set $X$ and every unitype rigid-based $X$-representable construction in $N, X$ is $X \cup Y$-uniformisable. So the hypothesis of Theorem 4.4 holds, and by that theorem there is in $N$ a small $\{\bar{c}\}$-uniformisable construction that is not weakly natural. But this contradicts the choice of $N$.

Problem $\mathrm{C}$ asked whether there are transitive models of ZFC in which every uniformisable construction is natural. Theorem 5.1 is the best answer we have for this; the problem remains open.

In [4] one of us asked whether there can be models of ZFC in which the algebraic closure construction on fields is not uniformisable.

Theorem 7.3. There are transitive models of ZFC in which:

(a) no formula without parameters defines for each field a specific algebraic closure for that field, and

(b) no formula without parameters defines for each abelian group a specific divisible hull of that group.

Proof. Let the model $N$ be as in Theorem 5.1. In $N$ the constructions of Example One in section 3 are not uniformisable, since they are not weakly natural. So these two examples prove (a) and (b) respectively.

We close with some remarks on related notions in other papers.

One result in [4] was that there is no primitive recursive set function which takes each field to an algebraic closure of that field. This is an absolute result which applies to every transitive model of ZFC, and so it is not strictly comparable with the consistency results proved above. In this context we note that Garvin Melles showed [8] that there is no "recursive set-function" (he gives his own definition for this notion) which finds a representative for each isomorphism type of countable torsion-free abelian group.

The paper [1] of Adámek et al. gives a simple universal algebraic sufficient condition for injective hull constructions not to be natural, and notes that two of their examples are also in 
[6]. The comparison between our notions and theirs is a little tricky. For both Adámek et al. and us, 'natural' is as in 'natural transformation' in the categorical sense. But we work in different categories. In this paper and [6], the relevant morphisms are isomorphisms; but for [1] they include embeddings. Hence the notion of naturality in [1] is stricter than ours. For example their condition implies that the MacNeille completion of posets, which embeds every poset in a lattice, is not natural. But it is natural in our sense, since isomorphisms between posets lift functorially to isomorphisms between their MacNeille completions. In fact this is clear from the standard definition of MacNeille completions ([2] p. 40ff), which also provides a uniformisation of this construction in any model of ZFC. It seems very unlikely that the condition in [1] adapts to give a sufficient condition for failure of weak naturality in the sense above.

In a related context Harvey Friedman [3] used the term 'naturalness' in a weaker sense than ours. 


\section{References}

[1] JiŘí Adámek, Horst Herrlich, JiŘí RosickÝ and Walter Tholen, 'Injective hulls are not natural', Algebra Universalis 48 (2002) 379-388.

[2] B. A. Davey and H. A. Priestley, Introduction to Lattices and Order, Cambridge University Press, Cambridge 1990.

[3] H. Friedman, 'On the naturalness of definable operations', Houston J. Math. 5 (1979) 325330.

[4] W. Hodges, 'On the effectivity of some field constructions', Proc. London Math. Soc. (3) 32 (1976) 133-162.

[5] W. Hodges, 'Definability and automorphism groups', in Proceedings of International Congress in Logic, Methodology and Philosophy of Science, Oviedo 2003, ed. Petr Hájek et al., King's College Publications, London 2005, pp. 107-120; ISBN 1-904987-21-4.

[6] W. Hodges and S. Shelah, 'Naturality and definability I', J. London Math. Soc. 33 (1986) $1-12$.

[7] T. JECH, Set theory (Academic Press, New York, 1978).

[8] G. Melles, 'Classification theory and generalized recursive functions', D.Phil. dissertation, University of California at Irvine, 1989. 